\title{
Tendências Epidemiológicas das Fraturas do Fémur Proximal na População Idosa em Portugal
}

\author{
Epidemiological Trends of Proximal Femoral Fractures \\ in the Elderly Population in Portugal
}

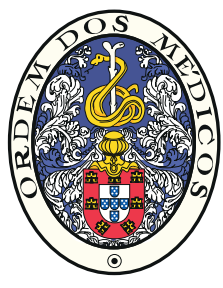

João SILVA ${ }^{1}{ }^{1}$, Daniela LINHARES ${ }^{1}$, Mariana FERREIRA ${ }^{1}$, Nélson AMORIM ${ }^{1}$, Nuno NEVES ${ }^{1}$, Rui PINTO1

Acta Med Port 2018 Oct;31(10):562-567 - https://doi.org/10.20344/amp.10464

\section{RESUMO}

Introdução: As fraturas do fémur proximal representam um problema de saúde pública nas sociedades industrializadas. São escassos os estudos sobre a incidência destas fraturas em Portugal. O objectivo deste estudo foi analisar e interpretar as tendências epidemiológicas das fraturas do fémur proximal, na população idosa em Portugal, no período de 2005 a 2013.

Material e Métodos: Estudo transversal incluindo todos os doentes com idade igual ou superior a 65 anos admitidos por fratura do fémur proximal em hospitais portugueses pertencentes ao serviço nacional de saúde com recurso aos dados do registo nacional da administração central do sistema de saúde.

Resultados: Foram incluídos 101436 doentes. Observou-se um aumento progressivo no número de internamentos por ano ao longo do período analisado. A idade média à admissão foi de 79,32 ( \pm 12.33$)$ anos apresentando um aumento progressivo e significativo ao longo do período analisado $(p<0,001)$. Dos doentes, $74.5 \%$ eram do sexo feminino $(p<0,001)$. Apresentavam idade média à admissão significativamente superior, quer globalmente, quer em cada ano em estudo $(p<0,001)$. A incidência ajustada à idade $(\geq 65$ anos) foi de 597 fraturas / ano / 100000 habitantes. Verificou-se um aumento na incidência de fraturas do fémur proximal de 508,49 (2005) para 628,39 fraturas por 100000 pessoas / ano (2013). No sexo feminino o aumento foi de 616,78 para 762,88 e no masculino de 339,95 para 419,06 .

Discussão: Este é o primeiro estudo a avaliar e interpretar as tendências epidemiológicas das fraturas do fémur proximal em doentes admitidos nos hospitais do Serviço Nacional de Saúde em Portugal incluindo apenas indivíduos com idade igual ou superior a 65 anos. Conclusão: O número absoluto de fraturas do fémur proximal em Portugal mostrou uma tendência de aumento entre 2005 - 2013 , afetando uma percentagem significativa da população portuguesa com mais de 65 anos de idade. Tendem a ocorrer mais comummente no sexo feminino e em indivíduos progressivamente mais velhos.

Palavras-chave: Fraturas do Colo Femoral/epidemiologia; Idoso; Osteoporose/complicações; Portugal

\section{ABSTRACT}

Introduction: Proximal femoral fractures are an important public health problem in industrialized societies. There are few studies that evaluate the incidence of this type of fracture in Portugal. The aim of this study was to analyze and interpret epidemiological trends of proximal femoral fractures in the elderly population in Portugal between 2005 and 2013.

Material and Methods: Cross-sectional study including all patients aged 65 years and older admitted due to proximal femoral fractures in Portuguese hospitals belonging to the National Health Service using data from the national registry of the health system central administration.

Results: 101,436 patients were included. There was a progressive increase in the number of hospitalizations per year over the period analyzed. The mean age at admission was $79.32( \pm 12.33)$ years presenting a progressive and significant increase over the analyzed period $(p<0.001) .74 .5 \%$ of the patients were female $(p<0.001)$. They had a significantly higher average age at admission, both globally and in each study year $(p<0.001)$. The age-adjusted incidence $(\geq 65$ years) was 597 fractures / year / 100,000 inhabitants. There was an increase in the incidence of proximal femoral fractures from 508.49 (2005) to 628.39 fractures per 100,000 person-years (2013). In the female sex the increase was from 616.78 to 762.88 and in the male sex from 339.95 to 419.06 .

Discussion: This is the first study to evaluate and interpret the epidemiological trends of proximal femur fractures in patients admitted to the National Health Service in Portugal including only individuals aged 65 years or over.

Conclusion: The global number of proximal femoral fractures in Portugal showed a trend of increase between 2005 - 2013, affecting a significant percentage of the Portuguese population over 65 years of age, especially in females and progressively older individuals. Keywords: Aged; Femoral Neck Fractures/epidemiology; Osteoporosis/complications; Portugal

\section{INTRODUÇÃO}

A osteoporose é uma doença prevalente nos idosos, estando frequentemente associada a um aumento do risco de fratura após traumatismo de baixa energia. Estudos recentes estimam que o número de fraturas osteoporóticas duplicará em 2040.1 As fraturas do fémur proximal (FFP) são uma das consequências mais graves da osteoporose, ${ }^{2}$ representando um importante problema de saúde pública nas sociedades ocidentais industrializadas. A taxa de mortalidade no primeiro ano após a fratura na população idosa pode atingir os $26 \% .^{3}$ As FFP estão associadas a um aumento da morbilidade e comprometimento funcional com impacto negativo na qualidade de vida dos doentes. ${ }^{2,4,5} \mathrm{~A}$ sua incidência está aumentada na população idosa, podendo condicionar a necessidade de institucionalização temporária ou definitiva. ${ }^{6,7}$

Diversos estudos mostram resultados divergentes na tendência da incidência das FFP. Um estudo Finlandês, mostrou uma estabilidade entre 1982 e 1993 mas, um

1. Serviço de Ortopedia e Traumatologia. Centro Hospitalar de São João. Porto. Portugal.

$\triangle$ Autor correspondente: João Silva. joao.duartesilva@hotmail.com

Recebido: 27 de fevereiro de 2018 - Aceite: 10 de julho de 2018 | Copyright @ Ordem dos Médicos 2018 
aumento significativo em ambos os géneros entre $1992 \mathrm{e}$ 2002. ${ }^{8}$ Outros estudos descrevem uma tendência de diminuição na ocorrência de FFP nos países do norte da Europa, América do Norte e Austrália. ${ }^{9-11}$ São escassos os estudos sobre a incidência deste tipo de fraturas em Portugal. Entre 2000 e 2008 foram registadas 77083 FFP, 77,4\% das quais em mulheres. ${ }^{12,13}$ Pina et al calcularam em 2007 as taxas de incidência anuais de FFP por região, obtendo valores compreendidos entre 154,4 e 572,2 nas mulheres e 77,4 e 231,5 nos homens, por cada 100000 habitantes. ${ }^{14}$

Tanto quanto sabemos não existem estudos em Portugal que ilustrem as tendências epidemiológicas das FFP na população geriátrica em Portugal.

O objetivo deste trabalho é descrever e analisar as tendências epidemiológicas das FFP, no período de 2005 a 2013, em indivíduos com idade igual ou superior a 65 anos admitidos em todos os hospitais portugueses pertencentes ao serviço nacional de saúde (SNS).

\section{MATERIAL E MÉTODOS}

Foi realizado um estudo transversal incluindo todos os doentes admitidos por FFP em hospitais do SNS. Através do registo nacional da administração central do sistema de saúde (ACSS), foram selecionados no período de 2005 a 2013 todos os doentes com idade igual ou superior a 65 anos que apresentavam como diagnóstico principal ou secundário/adicional o código 820 - Fratura do Colo do Fémur (GDH). A codificação foi baseada na modificação clínica da classificação internacional de doenças (CID) 9, ou seja, a ICD-9-CM, utilizada em todos os hospitais do SNS. Por dúvidas acerca da acuidade dos registos disponíveis nas regiões autónomas dos Açores e da Madeira, os doentes destas regiões foram excluídos da amostra.

\section{Análise estatística}

Os dados obtidos foram descritos usando tabelas de frequência. A análise dos dados foi realizada usando o teste $t$ de Student e seus equivalentes não paramétricos para variáveis contínuas e a análise de tabelas de contingência e o teste de qui-quadrado para variáveis categóricas. A normalidade das variáveis contínuas foi definida com base na análise visual do histograma. Foi usado o programa SPSS Statistics (Version 21.0). O cálculo da incidência das fraturas do fémur proximal na população estudada foi efetuado com recurso aos dados dos Censos de 2011.

\section{RESULTADOS \\ População}

A população portuguesa aumentou de 10,36 milhões em 2001 para 10,56 milhões em 2011, observando-se uma acentuação dos desequilíbrios demográficos com uma diminuição da base da pirâmide etária, correspondente à população mais jovem e um alargamento do topo, relacionado com o aumento relativo da população idosa. ${ }^{15} \mathrm{Na}$ última década a percentagem de jovens (população dos 0 - 14 anos) diminuiu de $16 \%$ em 2001 para 15\% em 2011, contrastando com o aumento da população de idosos (65 ou mais anos) de 16\% para 19\%. Em 2011 o índice de envelhecimento da população portuguesa aumentou para 128 (i.e. por cada 100 jovens há 128 idosos). ${ }^{15}$ Foram incluídos 101436 doentes, admitidos em todos os hospitais pertencentes ao SNS por FFP entre os anos de 2005 e 2013. Observou-se um aumento progressivo no número de internamentos por ano ao longo do período analisado, de 10221 doentes em 2005 para 12631 doentes em 2013 (Fig. 1). A idade média à admissão foi de 79,32 ( $\pm 12,33)$ anos com um aumento progressivo e significativo $(p<0,001)$ ao longo do período analisado (Fig. 2). $74,5 \%$ dos doentes eram do sexo

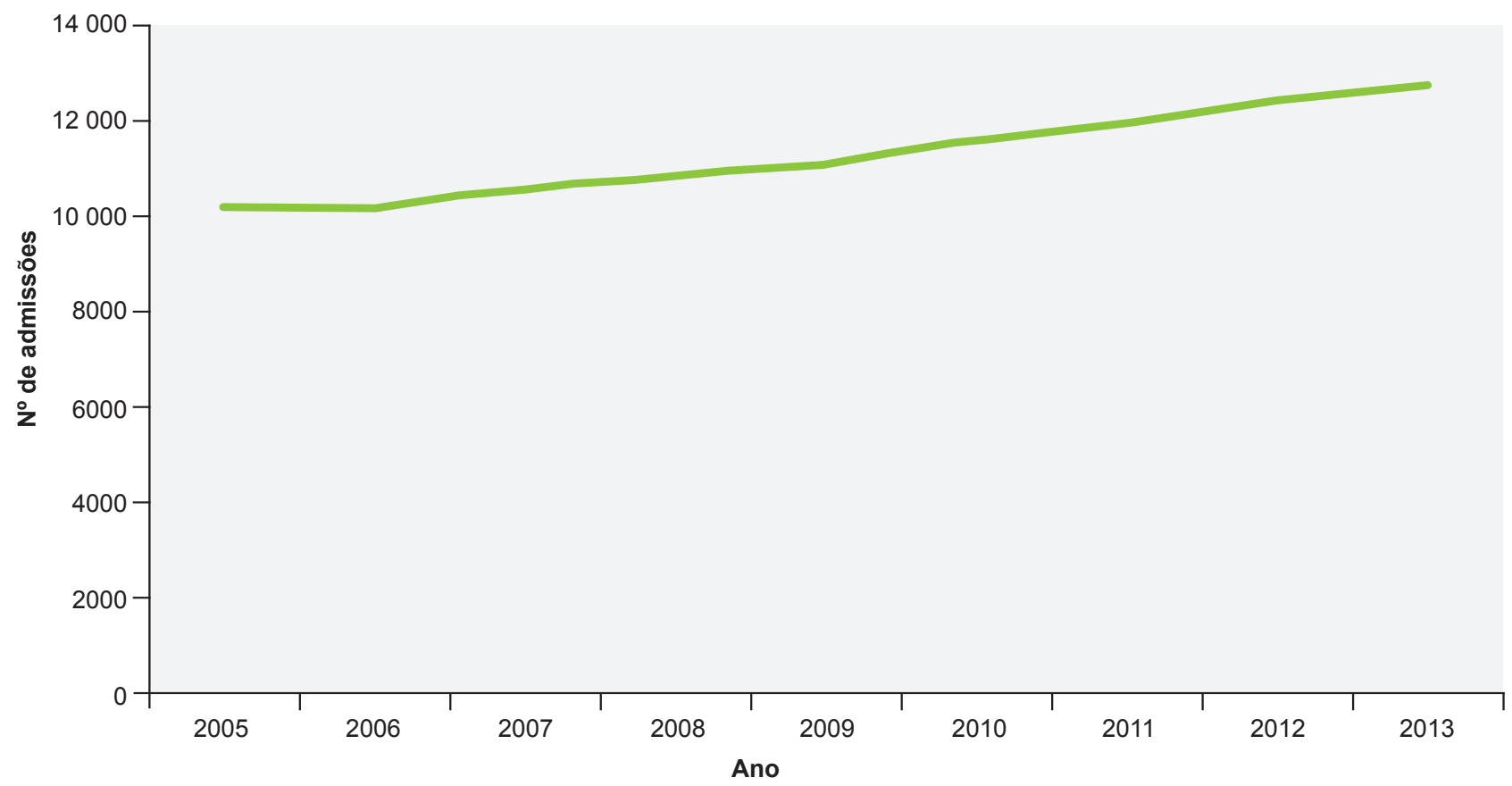

Figura 1 - Número de admissões de doentes de 2005 a 2013 


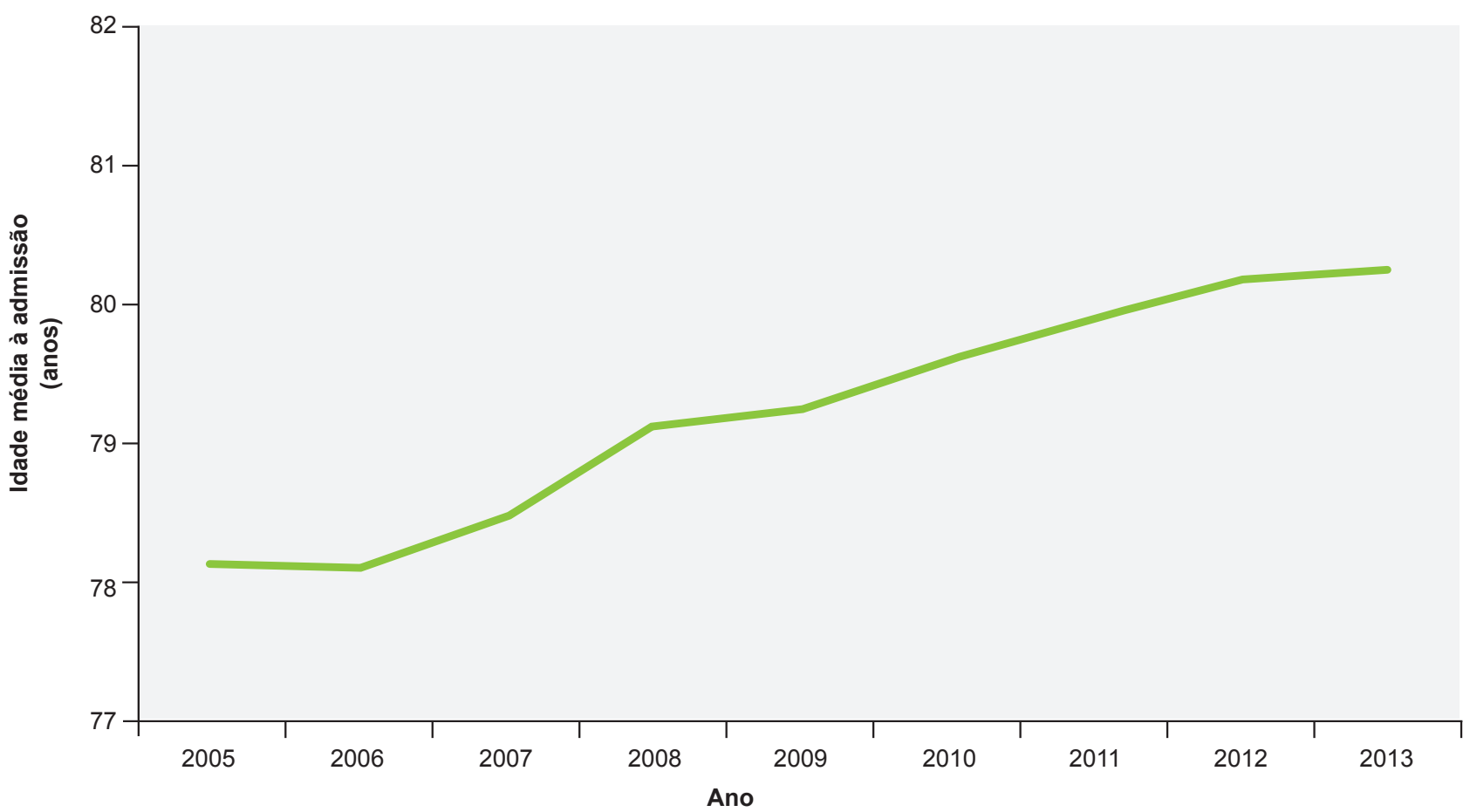

Figura 2 - Idade média dos doentes admitidos de 2005 a 2013

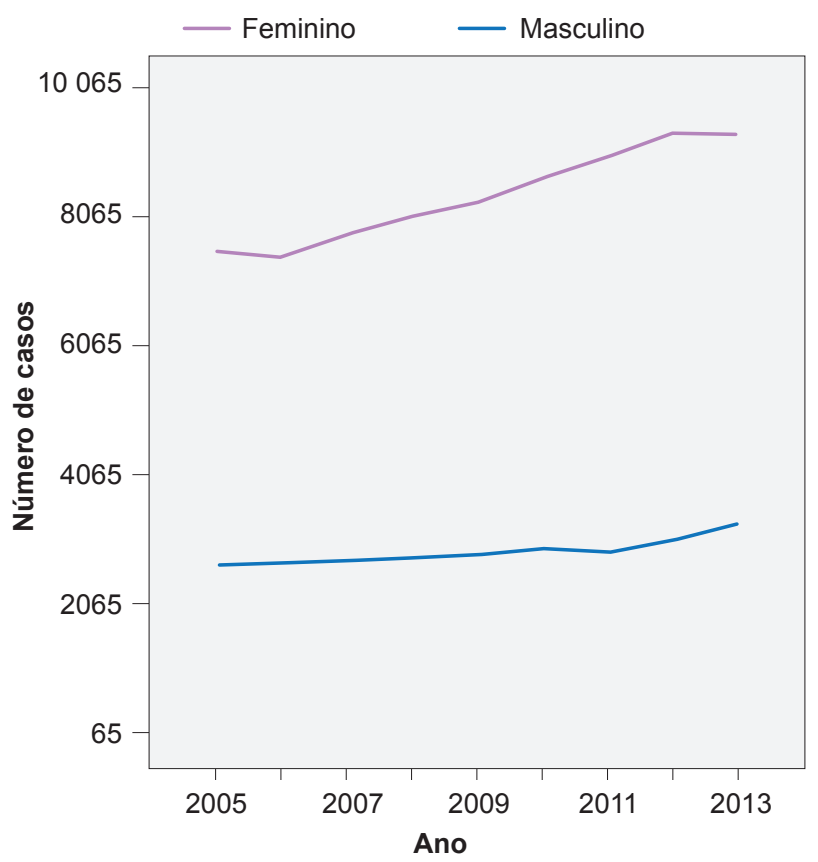

Figura 3 - Número de doentes admitidos por género de 2005 a 2013

feminino $(p<0,001)$, verificando-se uma tendência de aumento em ambos os sexos ao longo dos períodos de inclusão (Fig. 3). Os indivíduos do sexo feminino apresentavam uma idade média à admissão significativamente superior, quer globalmente, quer em cada ano em estudo $(p<0,001)$. Verificou-se uma tendência de aumento da idade média à admissão em ambos os sexos, sendo este aumento mais acentuado no sexo masculino (Fig. 4). A incidência ajusta-

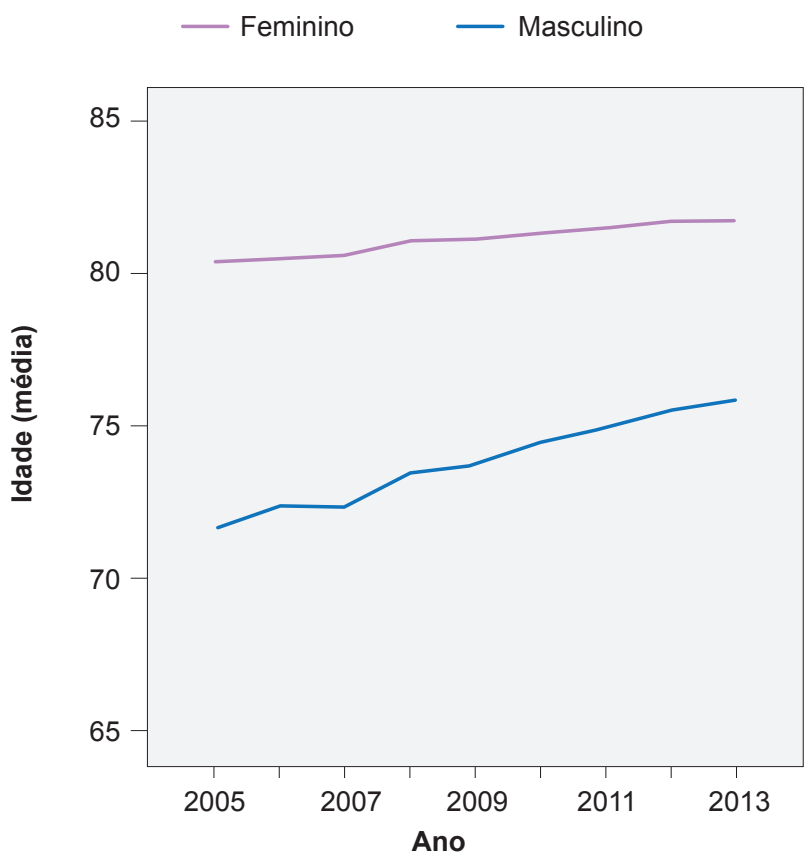

Figura 4 - Média de idade dos doentes admitidos por género de 2005 a 2013

da à idade ( $\geq 65$ anos) foi de 597 fraturas / ano / 100000 habitantes. Verificou-se um aumento na incidência de FFP de 508,49 em 2005 para 628,39 fracturas 100000 pessoas / ano em 2013 (Fig. 5). No sexo feminino o aumento foi de 616,78 para 762,88 e no sexo masculino de 339,95 para 419,06. (Fig. 6). O número médio de dias de internamento no ano de 2005 foi de 14,97 dias e de 14,5 dias em 2013. Em média os doentes permaneceram internados 14,77 


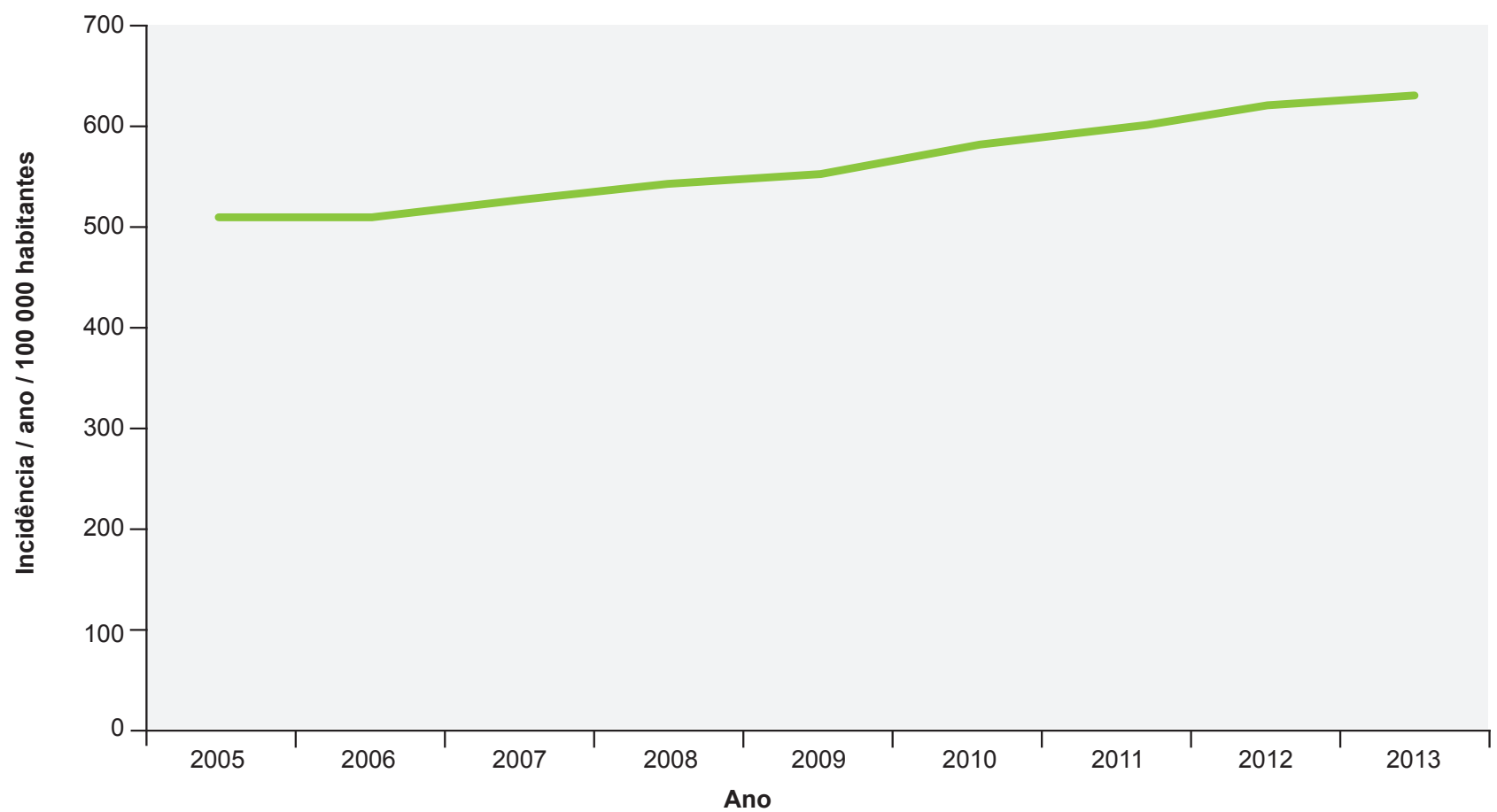

Figura 5 - Incidência de FFP ajustada à idade ( $\geq 65$ anos) de 2005 a 2013

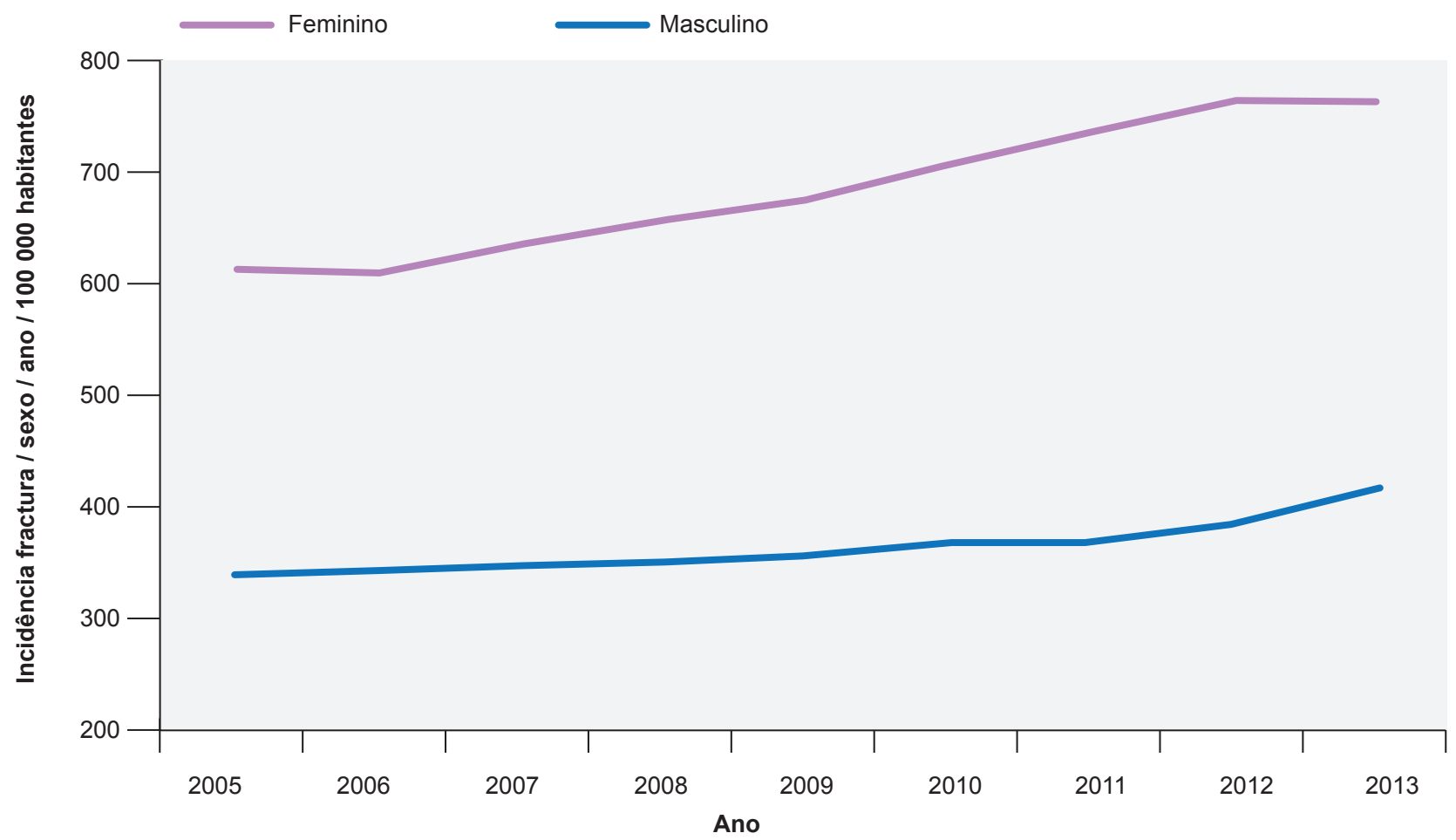

Figura 6 - Incidência de FFP ajustada à idade ( $\geq 65$ anos) e sexo de 2005 a 2013

$( \pm 18,95)$ dias. Ao longo do período do estudo observou-se uma variação no número médio de dias de internamento, sem uma tendência evidente, embora com diminuição no período mais recente em análise.

\section{DISCUSSÃO}

Este é o primeiro estudo a avaliar e interpretar as ten- dências epidemiológicas das FFP em doentes admitidos nos hospitais do SNS em Portugal incluindo apenas indivíduos com idade igual ou superior a 65 anos. Com o aumento da esperança média de vida e o envelhecimento da população, as FFP representam atualmente um dos principais problemas da traumatologia geriátrica, com importância significativa, quer na perspectiva do SNS, quer social. 
As FFP são uma das causas mais frequentes de hospitalização, representando cerca de metade dos motivos de internamento em serviços de ortopedia em indivíduos com $\geq$ 65 anos. ${ }^{16}$ Durante o período de nove anos do estudo (2005 a 2013) foram admitidos em Portugal continental 101436 doentes com FFP com $\geq 65$ anos, representando uma média de 11271 casos por ano. Verificou-se um aumento progressivo do número de internamentos por ano ao longo do período analisado, refletindo um aumento do número médio anual de fraturas.

As FFP tendem a ocorrer em indivíduos progressivamente mais velhos. Tal como previamente descrito na literatura, também em Portugal estas são significativamente mais frequentes no sexo feminino. ${ }^{17,18}$ São várias as explicações disponíveis para esta maior susceptibilidade, como a osteoporose após a menopausa, ${ }^{17}$ a maior expectativa de vida comparativamente ao sexo masculino e outros fatores genéticos relacionados com o género. ${ }^{19,20}$ Num estudo realizado em Inglaterra as taxas médias anuais de FFP em homens foram de 0,55 / 1000 e de 1,56 / 1000 em mulheres, ou seja, uma razão mulher/homem de $2,83 .{ }^{21} \mathrm{Um}$ outro estudo realizado na Coreia do Sul mostrou que $70,2 \%$ das FFP ocorreram em mulheres. ${ }^{22} \mathrm{Na}$ Noruega, um estudo realizado em quatro hospitais nos anos de 2004 e 2005, mostrou que $71,5 \%$ dos doentes eram do sexo feminino e $28,5 \%$ do sexo masculino. ${ }^{23}$ Estes resultados replicam o observado na população portuguesa.

Por outro lado, enquanto que na Dinamarca um estudo mostrou um aumento significativo na incidência de FFP em homens e mulheres, ${ }^{24}$ na Suécia, Löfman et al encontraram uma inversão da tendência de aumento na incidência de FFP em mulheres, mas não em homens. ${ }^{9} \mathrm{Na}$ Alemanha, Icks et al encontraram um aumento da incidência de FFP em homens, uma tendência de diminuição em mulheres até aos 74 anos, e um aumento significativo em ambos os géneros após os 74 anos de idade ${ }^{25}$. Na Suiça, Chevalley et al mostraram uma incidência estável de FFP em homens e uma diminuição significativa da incidência em mulheres. ${ }^{26}$ Contrariamente, os resultados em Portugal mostram uma tendência de aumento das FFP em ambos os géneros ao longo do período analisado. As diferenças no número de admissões por género podem dever-se quer a diferenças nos grupos etários avaliados, quer à implementação de programas de prevenção e tratamento da osteoporose em mulheres idosas. ${ }^{25,27}$

Quando avaliada a incidência ajustada à idade $(\geq 65$ anos) das FFP verificou-se um aumento de $12,4 \%$ entre 2005 e 2013, que derivará sobretudo do envelhecimento da população, comprovado nos dados já referidos dos últimos censos. ${ }^{15}$ Estudos publicados em outros países mostram resultados divergentes em relação às tendências da incidência das FFP. Assim sendo, Lönnroos et $a^{8}$ na Finlândia e Icks et $a^{25}$ na Alemanha mostram uma tendência de aumento de incidência das FFP, enquanto que Chevalley et $a l,{ }^{26}$ Chang et $a^{28}$ e Jaglal et $a^{29}$ na Suiça, Austrália e Canadá, respetivamente, mostram uma diminuição da mesma. Contudo, os dados destes estudos dizem respeito à população global, e não apenas ao subgrupo de indivíduos geriátricos, o que pode explicar a disparidade dos resultados quando comparados com a amostra portuguesa em estudo.

Este estudo, sendo multicêntrico, envolvendo todos os hospitais do SNS português e um período de inclusão de nove anos, permitiu a análise de um elevado número de casos, reforçando a validade dos resultados apresentados. As limitações do estudo prendem-se com o facto de ser um estudo transversal, com os vieses associados a possíveis erros de codificação e à ausência de validação interna e externa da mesma, bem como com o impacto da não análise de doentes com FFP não tratados em hospitais do SNS.

\section{CONCLUSÃO}

As FFP são objeto de estudo privilegiado, face às alterações demográficas associadas ao envelhecimento da população. O número absoluto de FFP em Portugal mostrou uma tendência de aumento entre 2005 - 2013, afetando uma percentagem significativa da população portuguesa com idade igual ou superior a 65 anos de idade. Estas tendem a ocorrer mais comummente no sexo feminino e em indivíduos progressivamente mais velhos. Estudos futuros devem avaliar as causas subjacentes às FFP e comparar os resultados apresentados com os de outros países. O tratamento farmacoterapêutico da osteoporose deve ser instituído assim como novas estratégias de prevenção devem ser procuradas, visando a diminuição da incidência das FFP. Uma melhor compreensão da sua epidemiologia é de suma importância, permitindo o desenvolvimento e implementação de políticas públicas preventivas favoráveis a esta população de doentes.

\section{PROTECÇÃO DE PESSOAS E ANIMAIS}

Os autores declaram que os procedimentos seguidos estavam de acordo com os regulamentos estabelecidos pelos responsáveis da Comissão de Investigação Clínica e Ética e de acordo com a Declaração de Helsínquia da Associação Médica Mundial.

\section{CONFIDENCIALIDADE DOS DADOS}

Os autores declaram ter seguido os protocolos do seu centro de trabalho acerca da publicação de dados.

\section{CONFLITOS DE INTERESSE}

Os autores declaram não terem qualquer conflito de interesse relativamente ao presente artigo.

\section{FONTES DE FINANCIAMENTO}

Os autores declaram não ter recebido subsídios ou bolsas para a elaboração do artigo. 


\section{REFERÊNCIAS}

1. Odén A, McCloskey EV, Kanis JA, Harvey NC, Johansson H. Burden of high fracture probability worldwide: secular increases 2010-2040. Osteoporos Int. 2015;26:2243-8.

2. Laires PA, Perelman J, Consciência JG, Monteiro J, Branco JC. Actualização sobre o impacto epidemiológico e socioeconomic das fracturas da extremidade proximal do femur. Acta Reumatol Port. 2015;40:223-30.

3. Klop C., Welsing PMJ, Cooper C, Harvey NC, Elders PJM, Bijlsma JWJ, Leufkens HGM, De Vries F. Mortality in British hip fracture patients, 2000-2010: A population-based retrospective cohort stud. Bone. 2014;66:171-7.

4. Keene GS, Parker MJ, Pryor GA. Mortality and morbidity after hip fractures. BMJ. 1993;307:1248-50.

5. Marques A, Lourenço Ó, da Silva JA: The burden of osteoporotic hip fractures in Portugal: costs, health related quality of life and mortality. Osteoporos Int. 2015;26:2623-30.

6. Cumming RG, Klineberg R, Katelaris A: Cohort study of risk of institutionalization after hip fracture. Aust $N$ Z J Public Health. 1996;20:579-82

7. Branco JC, Felicíssimo P, Monteiro J. A epidemiologia e o impacto socioeconomic das fracturas da extremidade proximal do fémur - uma reflexão sobre o padrão actual de tratamento da osteoporose grave. Acta Reumatol Port. 2009;34:475-85.

8. Lönnroos E, Kautianinen H, Karppi P, Huusko T, Hartikainen S, Kiviranta I, Sulkava R. Increased incidence of hip fractures. A population basedstudy in Finland. Bone. 2006;39:623-7.

9. Löfmann O, Berglund K, Larsson L, Toss G. Changes in hip fracture epidemiology: redistribution between ages, genders and fracture types. Osteoporos Int. 2002;13:18-25.

10. Jaglal SB, Weller I, Mamdani M, Hawker G, Kreder H, Jakkimainen L, Adachi JD. Population trends in BMD testing, treatment, and hip and wrist fracture rates: are the hip fracture projections wrong? J Bone Miner Res. 2005;20:898-905.

11. Chang KP, Center JR, Nguyen TV, Eisman JA. Incidence of hip and other osteoporotic fractures in elderly men and women: Dubbo Osteoporosis Epidemiology Study. J Bone Miner Res. 2004;19:532-6.

12. Alves SM, Castiglione D, Oliveira CM, de Sousa B, Pina MF. Age-periodcohort effects in the incidence of hip fractures: political and economic events are coincident with changes in risk. Osteoporos Int. 2014;25:71120.

13. Alves SM, Economou T, Oliveira C, Ribeiro Al, Neves N, GomézBarrena E, Pina MF. Osteoporotic hip fractures: Bisphosphonates sales and observed turning point in trend. A population-based retrospective study. Bone. 2013;53:430-6.

14. Pina MF, Alves SM, Barbosa M, Barros H. Hip fractures cluster in space: an epidemiological analysis in Portugal. Osteoporos Int. 2008;19:1797804.

15. Pordata Portugal. Censos 2011 - Resultados Definitivos. Fundação Francisco Manuel dos Santos. [Acedido em 2017 set 20]. Disponível em: http://www.pordata.pt/

16. Vanhaecht K, Sermeus W, Peers J, Lodewijckx C, Deneckere S, Leigheb $F$, et al. The impact of care pathways for patients with proximal femur fracture: rationale and design of a clusterrandomized controlled trial. BMC Health Serv Res. 2012;12:124

17. Guarniero R, Oliveira L. Osteoporose: atualização no diagnóstico e princípios básicos para o tratamento. Rev Bras Ortop. 2004;39:477-85.

18. Cummings SR, Melton LJ. Epidemiology and outcomes of osteoporotic fractures. Lancet. 2002;359:1761-7.

19. Einhorn TA. Bone strength: the bottom line. Calcif Tissue Int. 1992;51:333-9.

20. Villa ML, Nelson L, Nelson D. Race, ethnicity and osteoporosis. In: Marcus R, Feldman D, Kelsey J, eds. Osteoporosis, $2^{\text {nd }}$ ed. San Diego: Academic Press. 2001:569-84.

21. Wu TY, Jen MH, Garrafa A, Liaw CK, Aylin P, Majeed A. Admission rates and in-hospital mortality for hip fractures in England 1998 to 2009: time trends study. J Public Health. 2011;33:284-91.

22. Kang HY, Yang KH, Kim YN, Moon SH, Choi WJ, Kang DR, et al. Incidence and mortality of hip frac- ture among the elderly population in South Korea: a population-based study using the national health insurance claims data. BMC Public Health. 2010;10:230.

23. Diamantopoulos AP, Hoff M, Skoie M, Hochberg M, Haugeberg G. Shortand long-term mortality in males and females with fragility hip fracture in Norway: a population-based study. Clin Interv Aging. 2013;8:817-23.

24. Giversen IM. Time trends of age-adjusted incidence rates of first hip fractures: a register-based study among people in Viborg County, Denmark, 1987-1997. Osteoporos Int. 2006;17:552-64.

25. Icks A, Haastert B, Wildner C, Becker C, Meyer G: Trend of hip fracture incidence in Germany 1995-2004: a population based study. Osteoporos Int. 2008;19:1139-45.

26. Chevalley T, Guilley E, Herrmann FR, Hoffmeyer P, Rapin $\mathrm{CH}$, Rizzoli R: Incidence of hip fracture over a 10-year period (1991-2000): reversal of a secular trend. Bone. 2007;40:1284-9.

27. Johansson P, Sadigh S, Tillgren P, Rehnberg C: Non-pharmaceutical prevention of hip fractures - a cost-effectiveness analysis of a community-based elderly safety promotion program in Sweden. Cost Eff Resour Alloc. 2008;6:11 